Numerical Simulation of Interaction of Hypervelocity Particle Stream with a Target

I. Lomov, B. Liu, V. Georgevich, T. Antoun

August 14, 2007

Shock Compresion of Condensed Matter

Kona, HI, United States

June 23, 2007 through June 29, 2007 
This document was prepared as an account of work sponsored by an agency of the United States Government. Neither the United States Government nor the University of California nor any of their employees, makes any warranty, express or implied, or assumes any legal liability or responsibility for the accuracy, completeness, or usefulness of any information, apparatus, product, or process disclosed, or represents that its use would not infringe privately owned rights. Reference herein to any specific commercial product, process, or service by trade name, trademark, manufacturer, or otherwise, does not necessarily constitute or imply its endorsement, recommendation, or favoring by the United States Government or the University of California. The views and opinions of authors expressed herein do not necessarily state or reflect those of the United States Government or the University of California, and shall not be used for advertising or product endorsement purposes. 


\title{
NUMERICAL SIMULATION OF INTERACTION OF HYPERVELOCITY PARTICLE STREAM WITH A TARGET
}

\author{
Ilya Lomov, Benjamin Liu, Vlad Georgevich and Tarabay Antoun
}

Lawrence Livermore National Laboratory, Livermore CA 94550

\begin{abstract}
We present results of direct numerical simulations of impact of hypervelocity particle stream with a target. The stream of interest consists of submillimeter (30-300 micron) brittle ceramic particles. Current supercomputer capabilities make it possible to simulate a realistic size of streams (up to $20 \mathrm{~mm}$ in diameter and $500 \mathrm{~mm}$ in length) while resolving each particle individually. Such simulations make possible to study the damage of the target from synergistic effects of individual impacts. In our research we fixed the velocity distribution along the axis of the stream $(1-4 \mathrm{~km} / \mathrm{s})$ and volume fraction of the solid material (1-10\%) and study effects of particle size variation, particle and target material properties and surrounding air properties. We ran 3D calibration simulations with up to 10 million individual particles and conducted sensitivity studies with 2D cylindrically symmetric simulations. We used an Eulerian Godunov hydrocode with adaptive mesh refinement. The particles, target material and air are represented with volume-of-fluid approach. Brittle particle and target material has been simulated with pressure-dependent yield strength and Steinberg model has been used for metal targets. Simulations demonstrated penetration depth and a hole diameter similar to experimental observations and can explain the influence of parameters of the stream on the character of the penetration.
\end{abstract}

Keywords: Hypervelocity impact, direct numerical simulation, ceramic powder.

PACS: 46.15.-x, 47.11.Df.

\section{INTRODUCTION}

As computer capabilities grow, it becomes possible to simulate complex problems using direct numerical simulations of phenomena on the scales much lower then the overall problem size. This paper presents numerical simulation of such a problem, interaction of a hypervelocity particle stream with a target. This problem is particularly challenging because each individual impact is a highly nonlinear phenomenon with pressure spikes up to $1 \mathrm{Mbar}$, while average bow wave pressures are below 20 kbar. Simulation of individual impacts is very important since they induce damage of particles and target material and this process is very difficult to describe using homogenization techniques for heterogeneous and multiphase media.

\section{EXPERIMENTAL STUDIES}

Numerical simulations in this paper study conditions similar to experimental studies of a stream with velocity of 1 to $4 \mathrm{~km} / \mathrm{s}$ of $\mathrm{Al}_{2} \mathrm{O}_{3}$ ceramic particles impacting an aluminum target [1]. Simulations embark on an explanation of experimental observations listed below.

Experiments comparing the penetration of the particle stream in a vacuum with that in ambient air indicated relatively minor differences in penetration depth. However, the resulting hole was 


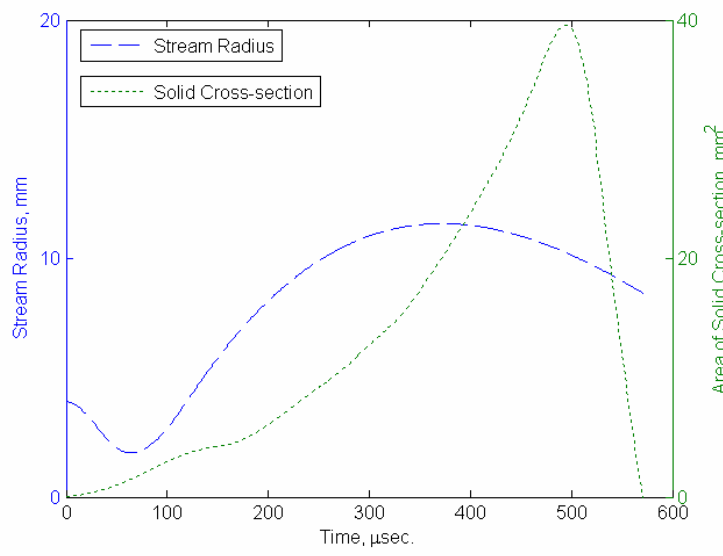

Figure 1. Particle stream radius and area of solid stream cross-section used in the simulation as boundary conditions.

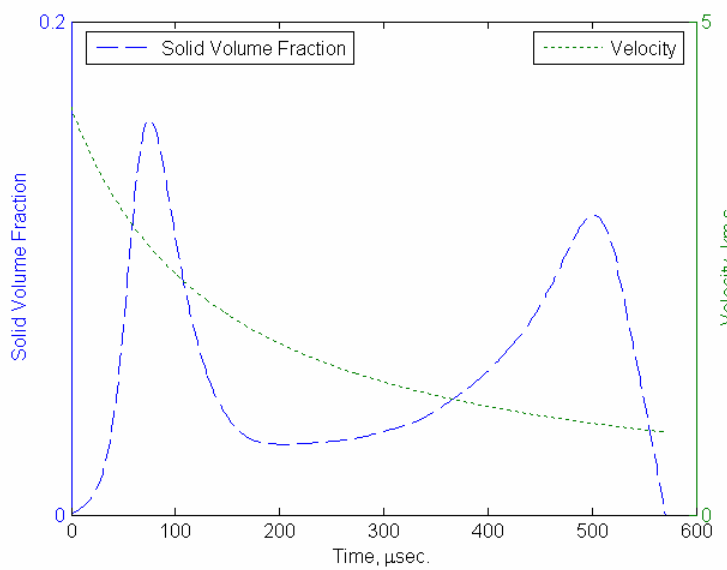

Figure 2. Solid volume fraction and velocity profiles used in the simulation as boundary conditions.

significantly cleaner (free of alumina) in the ambient air case.

Experiments show not monotonic dependence of penetration depth on particle size, but in general, streams with smaller particle sizes yield deeper holes.

We used X-ray snapshots and data from load cell experiments to derive boundary conditions for our simulations. Stream radius shown on Fig. 1is measured on a radiograph as a function of a stream position and converted to a function of time at the simulation boundary. The solid cross-section is derived from the load-cell data assuming inelastic capture. The stream has calculated solid volume fraction of 1-15\% (Fig 2). Velocity profile shown on Fig. 2 is calculated based on velocity measurement in several marked positions.

\section{GEODYN SIMULATIONS}

GEODYN, a multi-material Eulerian Godunov shock physics code [2] featuring material strength and adaptive mesh refinement (AMR) has been used for direct numerical simulation. GEODYN able to model large deformations of solids, capture shocks and track material interfaces with piecewise linear interface reconstruction (PLIC).

Particle alumina ceramic has been modeled with Mie-Gruneisen equation of state, pressuredependent yield strength and brittle damage with residual strength curve. We used random distribution of particles in space to match average experimental stream parameters on Figs. 1-2. Aluminum target has been modeled with MieGruneisen equation of state, Steinberg strength model with melting and ductile tensile damage.

\section{RESULTS AND DISCUSSION Mesh dependence}

Mesh dependence of the results turned out to be a big issue. Individual particles were resolved to $31.25 \mu \mathrm{m}$ in baseline simulations. We established that this resolution is appropriate to get correct characteristics (pressure, a crater size) for individual impacts. We could afford up to $4 \mathrm{x}$ higher resolution in $2 \mathrm{D}$ axisymmetric simulations. We found that penetration depth is mesh dependent, it can be up to $50 \%$ higher for a finer zone simulations. Possible explanation of this phenomenon can be explained by numerical surface tension introduced by the PLIC procedure. PLIC leads to coalescence of the small (comparable to the mesh size) pieces of aluminum into bigger agglomerates. So simulations with coarser mesh tend to have smaller interstitial area between ceramic and metal. This controls the shear strength of the ceramic-metal mixture in the hole. The stronger mixture is difficult to deform, hence it yields the shorter penetration depth.

While it is a serious issue which has to be resolved, it doesn't influence the qualitative results presented below 

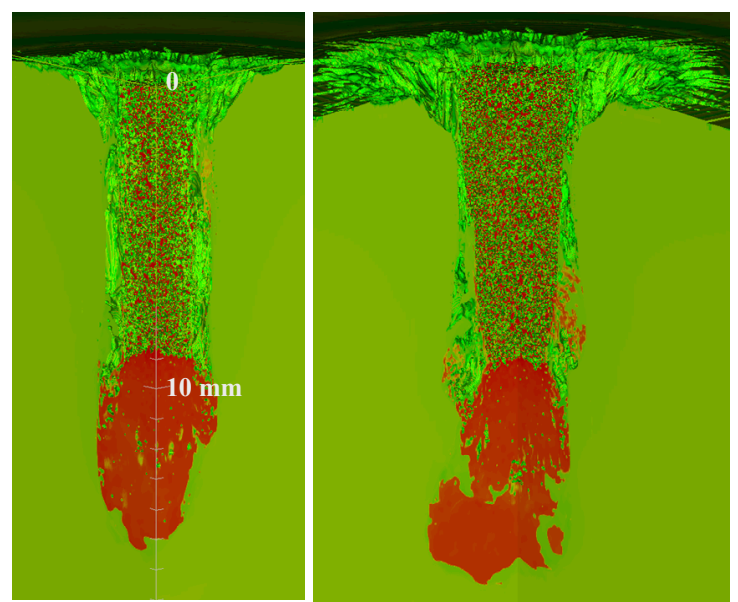

Figure 3. 3D Density Plots ( $1 / 4$ and $3 / 4$ cuts) after $75 \mu$ s of interaction of a $0.1 \mathrm{~mm}$ particle stream with a target.

\section{D Simulation}

3D simulation of a particle stream has been performed to establish validity of 2D axisymmetric used later for qualitative parametric studies. The mesh grew up to 100 million AMR cells. Major challenge has been the extremely small time step limited by the Courant condition. The simulation used 170,000 CPU hours for 400,000 timesteps. Fig. 3 shows snapshot of the simulation after $75 \mu$ s.

\section{D Study on Effect of Ambient Air}

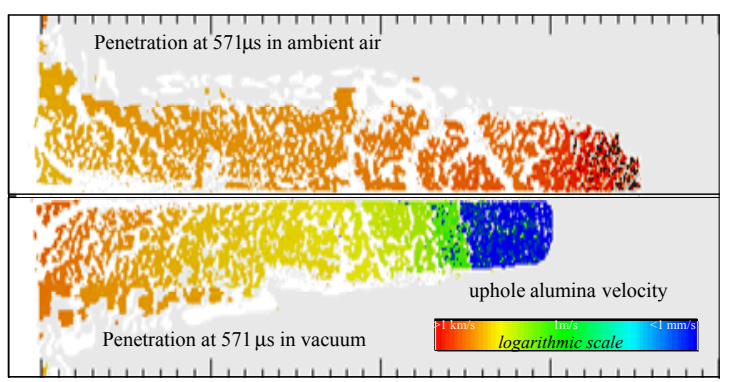

Figure 4. Simulations of a particle stream in a vacuum and in ambient air

Simulations of a particle stream in a vacuum and in ambient air showed similar penetration depths (within 20\%), demonstrating qualitative agreement with experiments. Ambient air was simulated as ideal gas $10^{-3} \mathrm{~g} / \mathrm{cc}$, vacuum was represented as rarified gas with density of $10^{-6} \mathrm{~g} / \mathrm{cc}$, similar to the experiment. The comparison of the velocities in the alumina indicates that air helps eject alumina from the hole (over time scales much longer than the penetration). This explains a clean hole for the experiment in ambient air and a slug in the hole for the experiment in vacuum.

\section{D Study on Particle Size Effects}

Axisymmetric 2D parameter studies were undertaken to determine the effects of particle size on penetration depth. Streams of 100 and 200 micron alumina particles in a vacuum were simulated, as well as a stream of continuum alumina (the limit of infinitesimal particles) and a series of alumina plates (a large particle limit). While the 200 micron particle stream penetrated further than the 100 micron particle stream, the continuum stream penetrated further than either. The alumina plates showed penetration in between the 100 and 200 micron cases. (Fig. 5)

This non-monotonic dependence on particle size is consistent with observations from experiments; further parameter studies are necessary to determine the correlation between particle size and penetration.

\section{CONCLUSIONS}

2D and 3D direct numerical simulations of ceramic particle stream penetration into a metal target have been performed. Mesh dependence and convergence is a major issue. Mesh size controls minimum droplet size, which influence strength of mixture

We found no major differences between $2 \mathrm{D}$ and 3D simumations, which makes it possible to perform the parametric studies in 2D setup. We found that ambient air doesn't change the character of the penetration but alters late time behavior. Also, penetration depth non-monotonically depends on particle size, similar to experimental observations.

\section{ACKNOWLEDGEMENTS}

This work was performed under the auspices of 
the U.S. Department of Energy by the University of California Lawrence Livermore National Laboratory under Contract No. W-7405-Eng-48.

\section{REFERENCES}

1. Baum, D. and J.B. Chase, Exploration of

Unconventional Properties of Ceramic Shaped-
Charge Jets, in Laboratory directed research and development FY98 annual report, R. Al-Ayat and J. Holzrichter, Editors. 1999.

2. Lomov, I. and M.B. Rubin, Numerical simulation of damage using an elastic-viscoplastic model with directional tensile failure. Journal De Physique Iv, 2003. 110: p. 281-286.

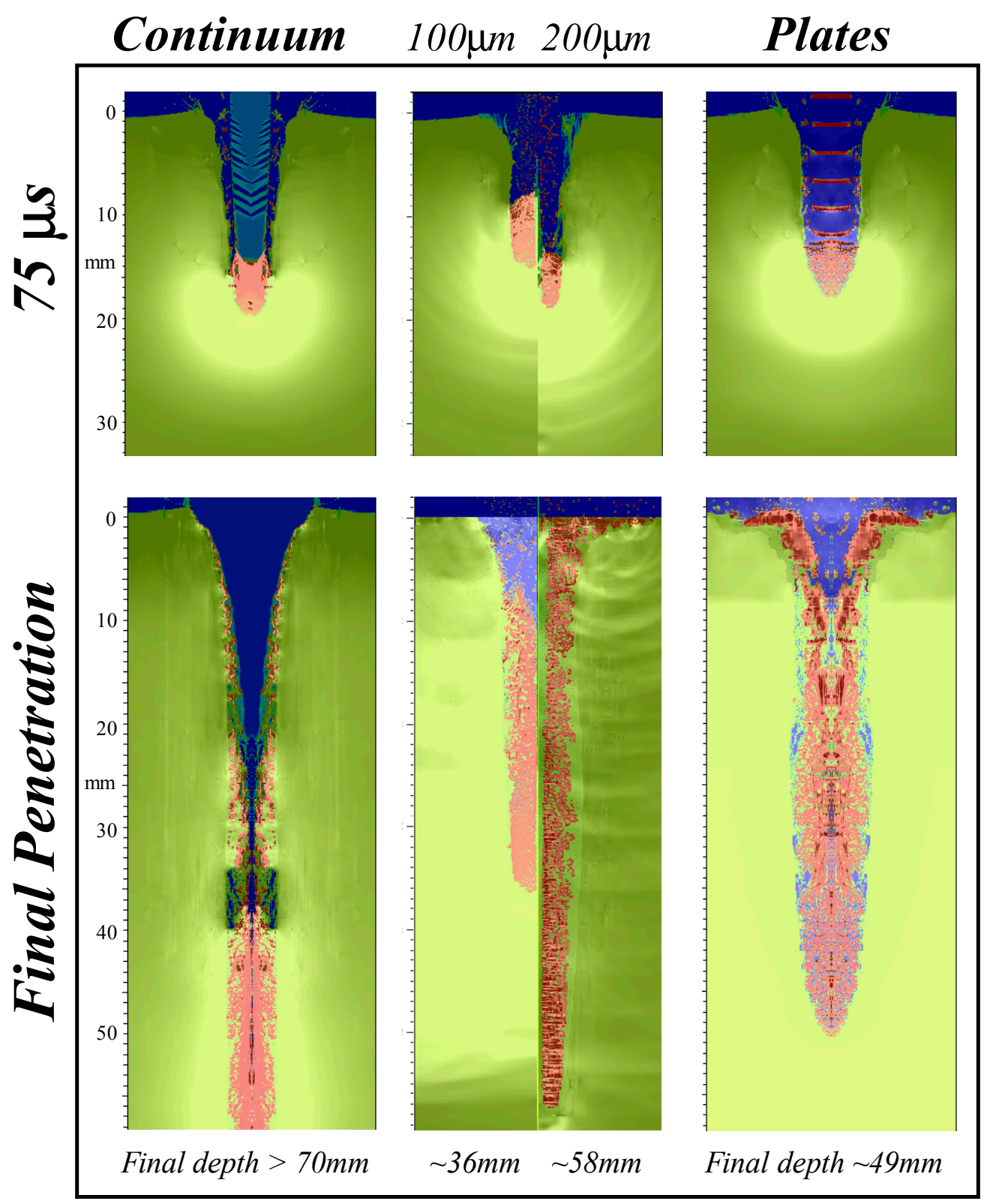

Figure 5. 2D Axisymmetric Study on Particle Size Effects 